\title{
Remarkably high mass and velocity dispersion of molecular gas associated with a regular, absorption-selected type I quasar
}

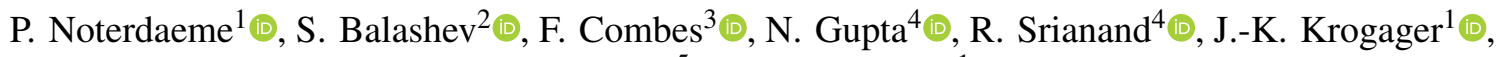 \\ P. Laursen ${ }^{5}$, and A. Omont ${ }^{1}(\mathbb{0}$ \\ ${ }^{1}$ Institut d'Astrophysique de Paris, CNRS-SU, UMR 7095, 98bis bd Arago, 75014 Paris, France \\ e-mail: noterdaeme@iap. fr \\ 2 Ioffe Institute, Polyteknicheskaya 26, 194021 Saint-Petersburg, Russia \\ 3 Observatoire de Paris, LERMA, Collège de France, CNRS, PSL University, Sorbonne University, 75014 Paris, France \\ ${ }^{4}$ Inter-University Centre for Astronomy and Astrophysics, Pune University Campus, Ganeshkhind, Pune 411007, India \\ 5 Cosmic Dawn Center (DAWN) and Niels Bohr Institute, University of Copenhagen, Jagtvej 128, 2200 Copenhagen N, Denmark
}

Received 8 March 2021 / Accepted 4 May 2021

\begin{abstract}
We present $3 \mathrm{~mm}$ observations of the quasar J0015+1842 at $z=2.63$ with the NOrthern Extended Millimeter Array (NOEMA) Our data reveal molecular gas, traced with a Gaussian $\mathrm{CO}(3-2)$ line, with a remarkably high velocity dispersion $(F W H M=1010 \pm$ $120 \mathrm{~km} \mathrm{~s}^{-1}$ ) that corresponds to a total molecular mass $M_{\mathrm{H}_{2}} \approx(3.4-17) \times 10^{10} M_{\odot}$, depending on the adopted CO-to- $\mathrm{H}_{2}$ conversion factor $\alpha_{\mathrm{CO}}=(0.8-4.0) M_{\odot}\left(\mathrm{km} \mathrm{s}^{-1} \mathrm{pc}^{2}\right)^{-1}$. Assuming the $3 \mathrm{~mm}$ continuum emission is thermal, we derive a dust mass of about $M_{\text {dust }} \sim 5 \times 10^{8} M_{\odot}$. J0015+1842 is located in the molecular gas-rich region in the IR versus CO line luminosity diagram, in between the main locus of main-sequence and sub-millimetre galaxies and that of most other active galactic nuclei targeted so far for $\mathrm{CO}$ measurements. While the high velocity dispersion of the CO line suggests a merging system, J0015+1842 is observed to be a regular, only very moderately dust-reddened $\left(A_{V} \sim 0.3-0.4\right)$ type I quasar from its UV-optical spectrum, from which we infer a mass of the super-massive black hole of about $M_{\mathrm{BH}} \approx 6 \times 10^{8} M_{\odot}$. We suggest that J0015+1842 is observed at a galaxy evolutionary stage where a massive merger has brought significant amounts of gas towards an actively accreting super-massive black hole (quasar). While the host still contains a large amount of dust and molecular gas with a high velocity dispersion, the quasar has already cleared the way towards the observer, likely through powerful outflows, as has recently been revealed by optical observations of the same object. High angular resolution observations of this and similar systems are expected to help us better determine the respective importance of evolution and orientation in the appearance of quasars and their host galaxies. These observations have the potential to investigate early feedback and star-formation processes in galaxies in their quasar phases.
\end{abstract}

Key words. radio lines: galaxies - galaxies: active - galaxies: evolution - quasars: individual: SDSS J001514.82+184212.34

\section{Introduction}

There is accumulating evidence for a strong link between the evolution of massive galaxies and the super-massive black hole $(\mathrm{SMBH})$ that they generally host in their centre (e.g., Heckman et al. 2004). When matter is accreted onto the disc surrounding the SMBH, enormous amounts of energy can be released through radiation (or relativistic jets), triggering the so-called galactic nuclear activity. Major mergers have been proposed as an efficient mechanism that causes the matter to lose most of its angular momentum and fall down to the inner regions of the galaxy on a relatively short timescale (e.g., Silk \& Rees 1998; Volonteri et al. 2003; Springel et al. 2005, but see Miki et al. 2021). At the same time, merging systems are known to induce intense star-formation activity through compression of the gas. Starburst galaxies and luminous unobscured active nuclei (quasars) might even be the same systems observed at different stages of the galaxy-SMBH co-evolution (Hopkins et al. 2008). In the rapid SMBH growth phase, huge amounts of dust and molecules are brought to the galaxy centre, and the SMBH activity remains heavily obscured. Bright, unobscured quasars would then correspond to a later phase, when the SMBH have almost fully assembled and are radiating close to their Eddington limit. The energy that is released could then be sufficient to significantly clear dust and gas from the entire galaxy through powerful winds. Such a feedback mechanism could be responsible for quenching star formation in the host (e.g., Zubovas \& King 2012; Pontzen et al. 2017; Terrazas et al. 2020), although the observed large-scale outflows may not necessarily arise from propagation of energy from the accretion disc to the interstellar medium (Fabian 2012; Veilleux et al. 2017), that is, be powered by activity from the active galactic nucleus (AGN), but might also result from the intense starformation activity or tidal ejection during the merging phase (e.g., Puglisi et al. 2021).

Because cold molecular gas is expected to support both the star-formation and the growth of the SMBHs, much effort has been devoted to studying the properties of this phase (e.g., Omont et al. 1996; Barvainis et al. 1997; Lewis et al. 2002; Bertoldi et al. 2003; Riechers et al. 2006; Weiß et al. 2007; Wang et al. 2010; Salomé et al. 2012 among many other works). In particular, many observing campaigns aimed at detecting the $\mathrm{CO}$ emission lines and constraining the molecular reservoirs of galaxies to investigate the evolutionary sequence between starburst (SB) galaxies and quasars, as well as AGN feedback (either positive or negative) on star formation in the host (e.g., 

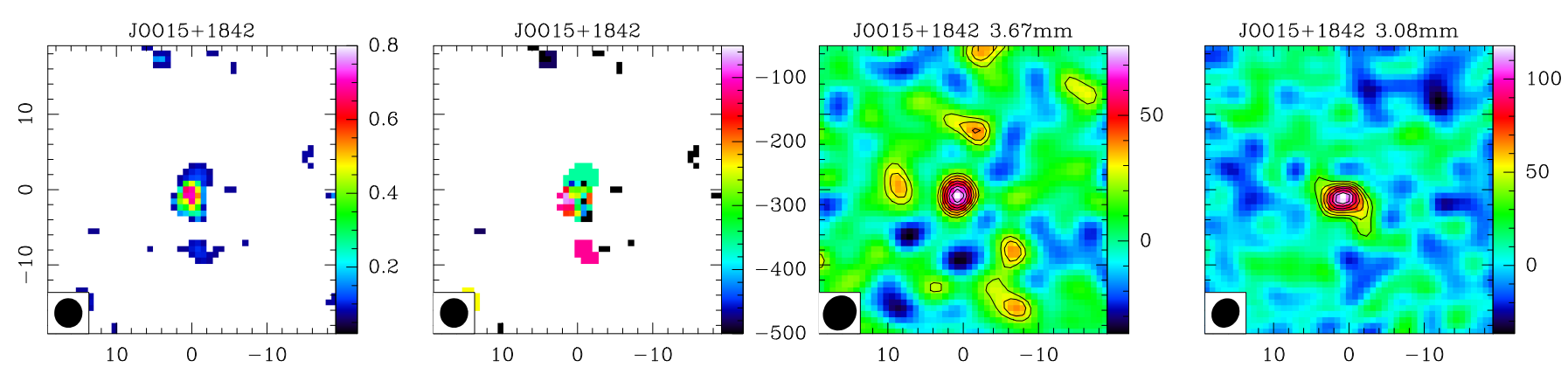

Fig. 1. From left to right: $\mathrm{CO}(3-2)$ integrated map, $\mathrm{CO}(3-2)$ velocity map, and continuum maps in the two sidebands (lower, $3.67 \mathrm{~mm}$, and upper, $3.08 \mathrm{~mm}$ ). The colour scale in each panel is in units of Jy beam ${ }^{-1} \times \mathrm{km} \mathrm{s}^{-1}, \mathrm{~km} \mathrm{~s}^{-1}$, and $\mu \mathrm{Jy}$ beam ${ }^{-1}$, respectively. The contours in the rightmost panels are drawn from 30 to $100 \%$ by $10 \%$ of the maximum, which is $78 \mu \mathrm{Jy}_{\text {beam }}{ }^{-1}$ and $118 \mu \mathrm{Jy} \mathrm{beam}^{-1}$. All axes are in offset arcseconds from the central position $\mathrm{RA}=00: 15: 14.81 \mathrm{Dec}=18: 42: 12.30$.

Weiß et al. 2012; Nesvadba et al. 2020). The studies have mostly focused on the bright end of the infra-red luminosity distribution and showed that luminous quasars generally have a low ratio of molecular gas masses to star formation rates (SFR) (e.g., Bischetti et al. 2021), while starburst galaxies have much higher ratios of molecular masses to SFR, supporting the evolutionary paradigm. Several studies have also searched for $\mathrm{CO}$ emission in obscured quasars, that is, possibly at the short-lived intermediate stage between the SB and optically bright quasar phase (e.g., Polletta et al. 2011; Brusa et al. 2015), but differences with luminous quasars are apparently found only for Compton-thick cases, which are more likely associated with the initial steps of the blow-out phase (Perna et al. 2018).

On the other hand, according to the AGN unification scheme (Antonucci 1993), the obscuration of a given AGN appears to depend on its orientation with respect to the observer, that is, it depends on whether the line of sight crosses large amounts of dust in the circum-nuclear region. It is therefore not absolutely clear how much the appearance of a system depends on its evolutionary stage and its orientation. It is also possible that the disc of the host galaxy significantly contributes to obscuring the nuclear region as well (e.g., Gkini et al. 2021).

Recently, Noterdaeme et al. (2019) uncovered a population of unobscured quasars in the Sloan Digital Sky Survey (SDSS York et al. 2000) that feature strong $\mathrm{H}_{2}$ absorption lines at the quasar redshift in their optical spectra. Detailed spectroscopic investigations of one of them, SDSS J001514.82+184212.34 (hereafter J0015+1842), with X-shooter on the Very Large Telescope suggested that the absorption system belongs to a galacticscale multi-phase outflow, which is also revealed by spatially resolved [O III] and Ly- $\alpha$ emission (Noterdaeme et al. 2021). In this Letter, we present the detection of strong and remarkably broad $\mathrm{CO}(3-2)$ emission with the NOrthern Extended Millimeter Array (NOEMA) in this quasar. We discuss these exceptional characteristics for an otherwise apparently regular quasar and suggest that the orientation of this system allows us to observe early AGN feedback during or after a merger phase.

\section{Observations}

Observations were carried out with NOEMA and the PolyFIX correlator in the $3 \mathrm{~mm}$ band in May and June, 2020. The $\mathrm{CO}(3-2)$ line at $z=2.631$ is redshifted at the $95.234 \mathrm{GHz}$ frequency. We observed in D configuration, with ten antennas on 23 and 29 May, and nine antennas on June 3. On May 23, the weather conditions were good and stable, and we obtained $2.4 \mathrm{~h}$ of integration. On May 29, most of the data were flagged because of poor weather, leaving $0.8 \mathrm{~h}$ of integration. On June 3, the weather was fine, providing us $4.9 \mathrm{~h}$ of integration time. Calibration was made using five sources, 3C345, 3C454.3, $0007+171,2010+723$, and MWC 349. The absolute flux calibration is accurate at the $10 \%$ level. The data were calibrated with the CLIC package and mapped with the MAPPING package in the GILDAS software ${ }^{1}$. We used CLARK cleaning in natural weighting, and the compact $\mathrm{D}$ configuration provided a beam of $3^{\prime \prime} .9 \times 3 \prime \prime 7$, with a PA of $69^{\circ}$ for the $\operatorname{CO}(3-2)$ line and the upper sideband continuum and $5^{\prime \prime} .1 \times 44^{\prime \prime} 5$, with a PA of $125^{\circ}$ for the lower sideband continuum. The continuum was also computed with the wider $7.7 \mathrm{GHz}$ upper sideband, with a beam of $3^{\prime \prime} .9 \times 3^{\prime \prime} .4$, with a PA of $-53^{\circ}$.

The quasar was observed in dual-polarisation mode in four basebands, with $3.9 \mathrm{GHz}$ total bandwidth per baseband, distributed in lower and upper sidebands distant by $15.5 \mathrm{GHz}$. The $\mathrm{CO}(3-2)$ line was observed in the upper sideband, and no other line was detected in the remaining basebands (upper and lower), which were used to estimate the continuum level. The velocity resolution was initially $2 \mathrm{MHz} \sim 6.29 \mathrm{~km} \mathrm{~s}^{-1}$. The spectra were then smoothed to $38.13 \mathrm{MHz}\left(120 \mathrm{~km} \mathrm{~s}^{-1}\right)$ to build channel maps. The final cube is $128 \times 128$ pixels with $0^{\prime \prime} .785$ per pixel in the plane of the sky, and has 80 channels of $120 \mathrm{~km} \mathrm{~s}^{-1}$ width. The noise level is $175 \mu \mathrm{Jy}$ beam ${ }^{-1}$ in $120 \mathrm{~km} \mathrm{~s}^{-1}$ channels for the line and $14 \mu \mathrm{Jy} \mathrm{beam}^{-1}$ for the continuum.

\section{Results}

Figure 1 presents the $\mathrm{CO}(3-2)$ integrated map, the velocity map, and the continuum maps in the two sidebands. The moments of the line cube were taken above a threshold of $3 \sigma$ in $120 \mathrm{~km} \mathrm{~s}^{-1}$ channels, corresponding to an integrated level of $0.063 \mathrm{Jy}$ beam $^{-1} \mathrm{~km} \mathrm{~s}^{-1}$.

\section{1. $\mathrm{CO}(3-2)$ emission}

The $\mathrm{CO}(3-2)$ emission is clearly detected in the integrated map, but the source remains unresolved, given the large beam size of $4^{\prime \prime}$, which corresponds to $32 \mathrm{kpc}$ at the quasar redshift. The firstmoment map of Fig. 1 presents a possible east-west velocity gradient, when the outflowing gas likely has a north-south direction projected on the sky (Noterdaeme et al. 2021).

In Fig. 2 we present the $3 \mathrm{~mm}$ spectrum extracted at the position of the quasar. We fitted the $\mathrm{CO}(3-2)$ line with a Gaussian function, yielding a total integrated flux $F_{\mathrm{CO}(3-2)}=1.1 \pm$ $0.2 \mathrm{Jy} \mathrm{km} \mathrm{s}^{-1}$, a full width at half maximum, $F W H M=1010 \pm$

1 http://wWw .iram.fr/IRAMFR/GILDAS/ 


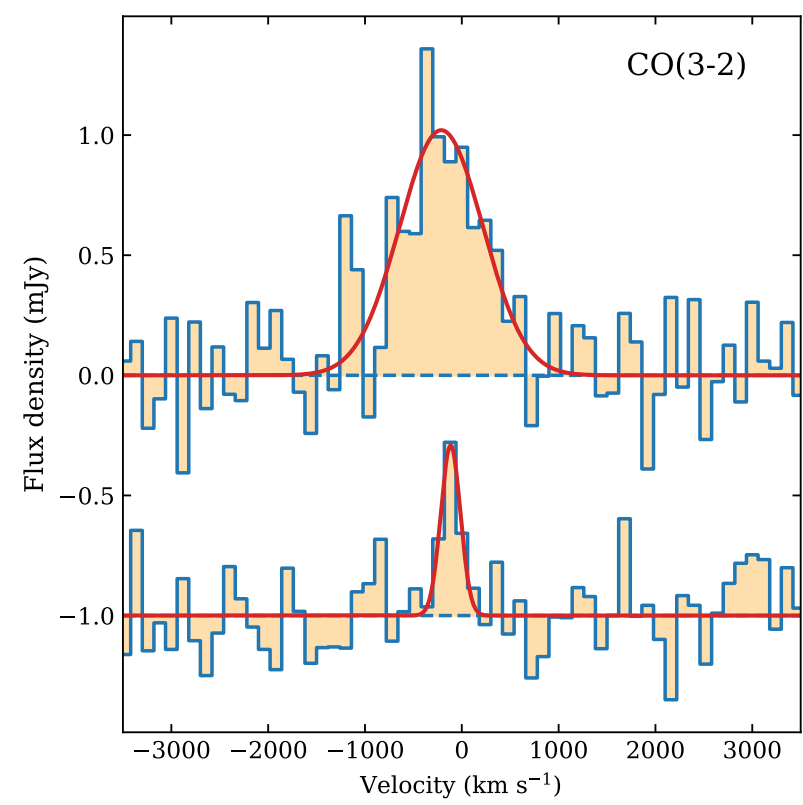

Fig. 2. $\mathrm{CO}(3-2)$ spectrum extracted using $120 \mathrm{~km} \mathrm{~s}^{-1}$ bins within a circle of 4 " diameter, i.e., an aperture as large as the synthesised beam of our NOEMA observations (top). The spectrum extracted at pixel $\left(-0.78,-7.85^{\prime \prime}\right)$ is also shown, shifted by $-1 \mathrm{mJy}$ for convenience. The zero of the velocity scale corresponds to the systemic redshift $z=2.631$ inferred from the narrow [O III] emission (Noterdaeme et al. 2021).

$120 \mathrm{~km} \mathrm{~s}^{-1}$, and a central velocity $v=-210 \pm 50 \mathrm{~km} \mathrm{~s}^{-1}$ with respect to the systemic redshift ${ }^{2}$. This shift, if real, may be due to blending of $\mathrm{CO}$ lines in a complex system. We converted the flux into luminosity following Solomon \& Vanden Bout (2005) and obtained $L_{\mathrm{CO}(3-2)}^{\prime} \approx 4.1 \times 10^{10} \mathrm{~K} \mathrm{~km} \mathrm{~s}^{-1} \mathrm{pc}^{2}$. To estimate the mass of molecular gas from the $\mathrm{CO}$ line luminosity, we assumed a typical $\mathrm{CO}$ spectral line energy distribution for quasars and took the $\mathrm{CO}(3-2) / \mathrm{CO}(1-0)$ intensity ratio $r_{31}=0.97$ from Carilli \& Walter (2013), consistent with observations of AGN host galaxies at $z>2$ (Sharon et al. 2016). The CO-to- $\mathrm{H}_{2}$ conversion factor depends on the average conditions in the molecular gas, which we do not know. We hence obtain a conservative range $M_{\mathrm{H}_{2}}=\alpha_{\mathrm{CO}} L_{\mathrm{CO}(1-0)}^{\prime} \approx(3.4-17) \times 10^{10} M_{\odot}$ assuming $\alpha_{\mathrm{CO}}=0.8 M_{\odot}\left(\mathrm{km} \mathrm{s}^{-1} \mathrm{pc}^{2}\right)^{-1}$ (as generally adopted for quasars; Walter et al. 2003; Wang et al. 2010; Bolatto et al. 2013) and $\alpha_{\mathrm{CO}}=4 M_{\odot}\left(\mathrm{km} \mathrm{s}^{-1} \mathrm{pc}^{2}\right)^{-1}$ (standard value).

Finally, we also note a possible structure about $8^{\prime \prime}(\sim 60 \mathrm{kpc}$ at $z=2.631)$ southwards of the quasar in both the zero- and first-moment maps. This could be due to a gas-rich companion galaxy that is not seen in the optical. The corresponding extracted spectrum has $F_{\mathrm{CO}(3-2)}=0.18 \pm 0.06 \mathrm{Jy} \mathrm{km} \mathrm{s}^{-1}$, $F W H M=240 \pm 60 \mathrm{~km} \mathrm{~s}^{-1}$, and $v=-115 \pm 30 \mathrm{~km} \mathrm{~s}^{-1}$. This corresponds to a molecular mass of $2.8 \times 10^{10} M_{\odot}$, assuming the same $r_{31}$ as above and the standard $\alpha_{\mathrm{CO}}$. Deeper observations with higher spatial resolution are necessary to better unveil the quasar and its environment.

\subsection{Continuum $3 \mathrm{~mm}$ emission and FIR luminosity}

We detect the $3 \mathrm{~mm}$ continuum emission at the position of the quasar on the map with a flux density, determined by fitting uv visibilities, of $65 \pm 14 \mu \mathrm{Jy}$ and $104 \pm 14 \mu \mathrm{Jy}$ for the lower

${ }^{2} z=2.631$, inferred with $\sim 100 \mathrm{~km} \mathrm{~s}^{-1}$ uncertainty from the narrow [O III] emission.

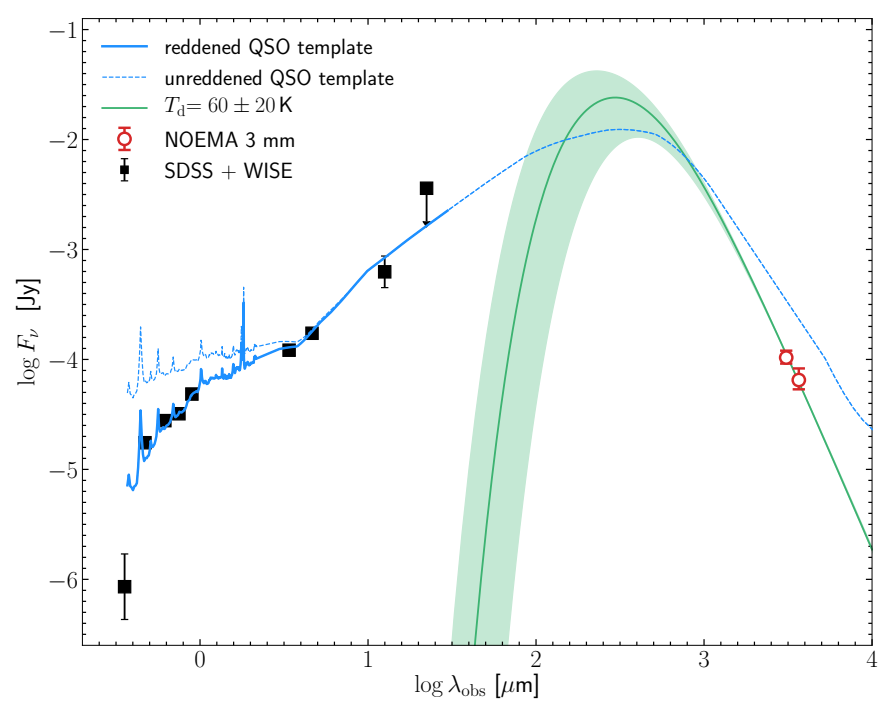

Fig. 3. Fit to the spectral energy distribution of the quasar J0015+1842 using photometric points from SDSS and WISE (black squares). The solid (resp. dotted) blue line shows the reddened (resp. unreddened) quasar type I template from Polletta et al. (2007). The NOEMA continuum measurements at 3.08 and $3.67 \mathrm{~mm}$ are represented by the red circles with error bars, and the associated constraint on the cold dust emission component assuming $T_{\mathrm{d}}=60 \pm 20 \mathrm{~K}$ is shown in green.

$(81.76 \mathrm{GHz}$ or $3.67 \mathrm{~mm})$ and upper $(97.24 \mathrm{GHz}$ or $3.08 \mathrm{~mm})$ sidebands, respectively. The flux density varies with frequency as a power law with slope of $v^{\alpha}$ with $\alpha=2.7$, that is, it is consistent with dust emission, with a dust opacity varying as $\nu^{\beta}$, with $\beta=0.7$, but with a large $( \pm 1.2)$ statistical uncertainty ${ }^{3}$. Following Carniani et al. (2017 their Eq. (2)), but assuming a dust temperature in the range $40-80 \mathrm{~K}$ and $\beta$ within the range obtained above, we infer a dust mass of $M_{\text {dust }} \approx 5 \times 10^{8} M_{\odot}$, within a factor of two, assuming thermal emission alone.

We constrained the AGN spectral energy distribution (SED) from the fluxes measured in SDSS and Wide-field Infrared Survey Explorer (WISE, Wright et al. 2010) filters ${ }^{4}$, using the template by Polletta et al. (2007), only moderately reddened by dust ( $A_{V} \sim 0.3$, consistent with $A_{V}=0.4 \pm 0.1$ derived from the $\mathrm{X}$-shooter spectrum and template, Noterdaeme et al. 2021), see Fig. 3. Based on this template, the intrinsic bolometric luminosity is found to be $\log L_{\mathrm{bol}} / L_{\odot} \simeq 13.4$. We note that the relative contribution of the host galaxy is probably different than predicted by this template, which over-predicts the $3 \mathrm{~mm}$ continuum emission. We fitted this emission using a modified blackbody emission law (see e.g., Eq. (2) from Rangwala et al. 2011), as expected for reprocessed cold dust emission. Using standard values for a power-law slope $(\beta=1.6$, consistent with our constraint) and normalisation point of the frequency dependence of the effective optical depth $\left(v_{0}=1.5 \mathrm{THz}\right)$, and assuming a dust temperature $T_{\mathrm{d}}=60 \mathrm{~K}$, we obtain a total infra-red luminosity associated with the host $\log L_{\mathrm{IR}} / L_{\odot} \sim 12.7$. We caution, however, that this estimate is very uncertain in the absence of measurements at $100-1000 \mu \mathrm{m}$ to constrain $T_{\mathrm{d}}$ and $\beta$. When we

\footnotetext{
3 Systematics are expected to be much lower than this value because the relative flux calibration of the basebands (USB and LSB) of NOEMA is very good (Neri et al. 2020).

4 The $u$ band is not considered because it is affected by Ly- $\alpha$ forest and $\mathrm{H}_{2}$ absorption. W4 provides an upper limit. The quasar presents only $\sim 0.25 \mathrm{~V}$-mag variability according to the Catalina Sky Survey (Drake et al. 2009), consistent with the long-term variability of other
} quasars (Hook et al. 1994). 


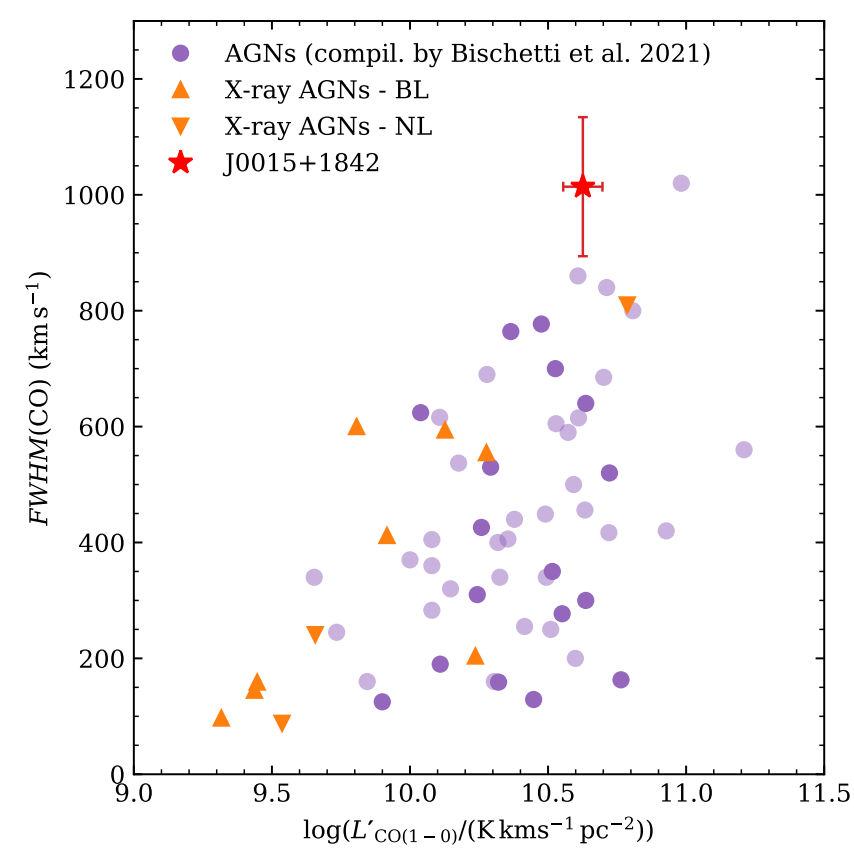

Fig. 4. Full width at half maximum vs. luminosity of the CO emission line in J0015+1842 (red star) compared to that in $z>1$ AGNs (compiled by Bischetti et al. (2021), with X-ray selected broad-line (BL) and narrow-line (NL) AGNs from Circosta et al. (2021) shown by orange triangles). Measurements using $\mathrm{CO}(3-2)$ are shown with darker symbols than those from other $\mathrm{CO}$ lines.

assume $T_{\mathrm{d}}$ in the range $40-80 \mathrm{~K}$, this results in an uncertainty of about one order of magnitude of $\log L_{\mathrm{IR}} / L_{\odot}=12.2-13.1$. We also note that if a merging or companion galaxy contributes to this infra-red luminosity (Bischetti et al. 2021), then the value could be considered as an upper limit to the infra-red luminosity of the quasar host alone.

\section{Discussion and conclusions}

In order to maximise the chances of detection, the vast majority of $z>1$ AGNs observed so far in CO lines have been selected for being among the brightest objects, in particular, at millimetre or infra-red wavelengths (e.g., Coppin et al. 2008; Wang et al. 2010, 2013, 2016; Simpson et al. 2012; Feruglio et al. 2014; Fan et al. 2019; Banerji et al. 2021). For example, WISE has played an important role in selecting these hyperluminous objects, in combination with (e.g., Bischetti et al. 2017) or without (Fan et al. 2018) photometry from the SDSS. Other works focused on possibly less luminous objects that were still preferentially dust obscured (e.g., Polletta et al. 2011; Banerji et al. 2017; Kakkad et al. 2017; Perna et al. 2018), radio loud (e.g., Willott et al. 2007), or on the highest redshifts detected in [C II] $\lambda 158 \mu \mathrm{m}$ (e.g., Venemans et al. 2017). In turn, J0015+1842 is not particularly bright, with an $i$-band magnitude in only the second brightest quartile of SDSS quasars at the same redshift, for instance, and it is only moderately reddened $\left(A_{V} \sim 0.3-0.4\right)$. It also has $\sim 3 \mathrm{mag}$ fainter WISE magnitudes than those of WISSH quasars (Bischetti et al. 2017).

In Fig. 4 we compare the width and luminosity of the detected $\mathrm{CO}$ line with those seen in other $z>1$ AGNs from the literature. We used the compilation of unlensed AGNs by Bischetti et al. (2021) and retrieved the line widths from the original detection publications. In a few cases where several spectrally resolved components were reported, we

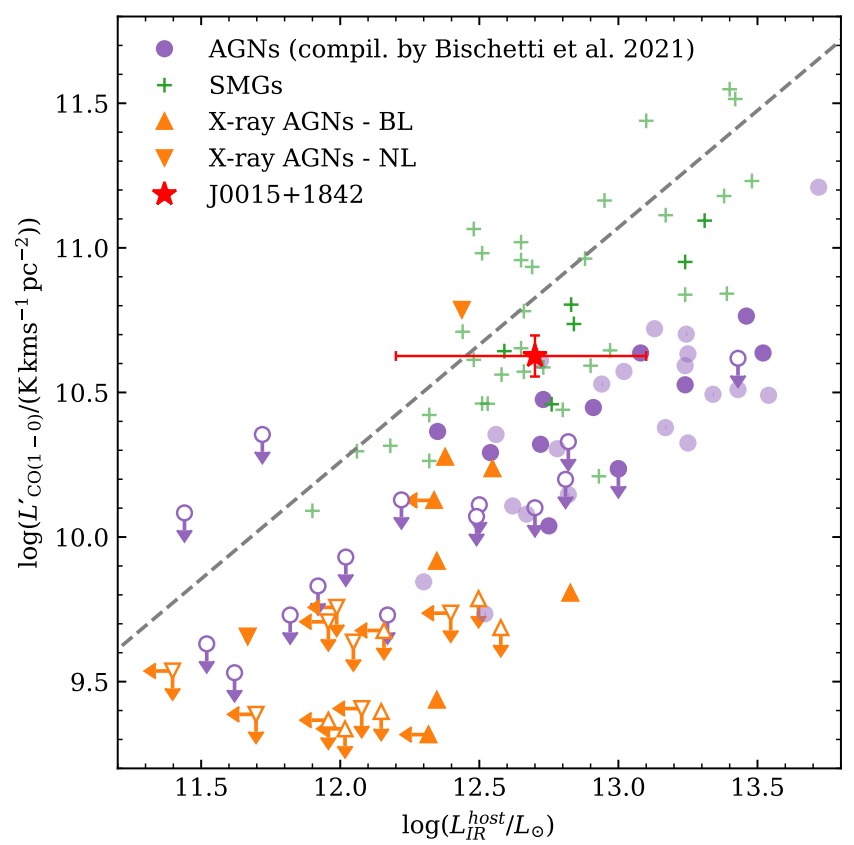

Fig. 5. CO line luminosity against the infra-red luminosity of the host integrated in the $8-1000 \mu \mathrm{m}$ range. Empty symbols correspond to $\mathrm{CO}$ upper limits in case of non-detection, and green crosses show submillimetre galaxies. Other symbols are the same as in Fig. 4. The dashed line corresponds to the relation for main-sequence galaxies (Sargent et al. 2014).

conservatively considered the widest component. To avoid systematics related to the assumption of CO-to- $\mathrm{H}_{2}$ conversion factor, we compare directly the $\mathrm{CO}$ line luminosity instead of the $\mathrm{H}_{2}$ mass. This compilation also includes the sample by Circosta et al. (2021), who recently performed an X-ray selection of both type I and type II AGNs with a range of bolometric luminosities, independently of their millimetre or infra-red emission. This more homogeneous sample also presents the advantage of being observed entirely in the $\mathrm{CO}(3-2)$ line, so that a direct comparison can be made with $\mathrm{J} 0015+1842$ regardless of the assumption of the excitation correction. We therefore distinguish this sample from other AGNs in Figs. 4 and 5.

The luminosity and line width of J0015+1842 are remarkably high, and the width measured here, FWHM $=1010 \pm$ $120 \mathrm{~km} \mathrm{~s}^{-1}$, is well above the vast majority of other measurements in the compiled sample, only equated by the powerful obscured quasar SWIRE J022513-043419, which has FWHM $\approx$ $1020 \pm 110 \mathrm{~km} \mathrm{~s}^{-1}$ (Polletta et al. 2011). Like J0015+1842, the latter object also presents extended [O III] emission that may trace outflowing gas (Nesvadba et al. 2011).

We compared the CO content of J0015+1842 with that of other AGNs as a function of the infra-red luminosity in Fig. 5 using the same compilation as previously, but restricted to systems for which Bischetti et al. (2021) considered the infra-red measurement to be reasonably reliable (their Sect. 6.2). We also add sub-millimetre galaxies (SMGs) to the comparison. The infra-red luminosity, integrated over the range $8-1000 \mu \mathrm{m}$, is generally considered as a proxy of dust emission related to star formation in the host when the AGN contribution has been removed. The infra-red-to-CO luminosity ratio is then a widely used proxy for star formation efficiency. Remarkably, the high CO-line luminosity of J0015+1842 is similar to that of the most luminous AGNs in the infra-red. However, with its likely lower cold dust emission, J0015+1842 is 
located closer to the region populated by MS galaxies and SMGs, and with molecular content well above that of most X-ray selected AGNs. The only AGN in the sample of Circosta et al. (2021) with similar CO luminosity is cid_1253 (COSMOS $\mathrm{J} 100130.56+021842.6)$ with $\log \left(L_{\mathrm{CO}(3-2)}^{\prime} /\left(\overline{\mathrm{K}} \mathrm{km} \mathrm{s}^{-1} \mathrm{pc}^{-2}\right)\right)=$ $10.80 \pm 0.04$ and $F W H M(\mathrm{CO})=810 \pm 93 \mathrm{~km} \mathrm{~s}^{-1}$. This object is a merger, hosting a narrow-line (type II) AGN, while J0015+1842 is a regular quasar with broad emission lines (type I).

According to the evolutionary scheme, major mergers of gas-rich galaxies trigger starburst activity and bring a significant amount of matter towards the nuclear regions, initiating the build-up of the supermassive black hole. These objects would mostly appear first as obscured AGNs, with star formation efficiencies much higher than in main-sequence galaxies, and would then transition to optically bright systems with low gas fractions. The detection of powerful multi-phase galactic-scale outflows shows that AGNs are capable of removing large amounts of gas from their host galaxies. As recently discussed by Perna et al (2018), neither the evolutionary sequence nor orientation effects with respect to the obscuring medium alone are able to explain the observed SFEs in obscured and unobscured AGNs.

$\mathrm{J} 0015+1842$ is bringing an important piece to this puzzle. The exceptionally broad Gaussian profile of the $\mathrm{CO}$ line in J0015+1842 suggests a recent or ongoing merger, with a very significant amount of molecular gas and dust. However, the line of sight to the active nucleus is only very moderately reddened and the presence of broad emission lines corresponds to a regular type I quasar powered by a highly accreting SMBH with an Eddington ratio ${ }^{5} \lambda_{\mathrm{Edd}}=L_{\mathrm{bol}} / L_{\mathrm{Edd}} \gtrsim 1$.

From the analysis of ionised emission lines together with absorption from $\mathrm{H}_{2}$, Noterdaeme et al. (2021) suggested that a multi-phase outflow is observed, oriented almost towards the observer. This could provide a natural explanation to the low extinction along the line of sight. J0015+1842 would therefore support a picture in which feedback processes can start early in the evolutionary sequence, with outflows clearing the view towards the nuclear region at least in some directions, while a large amount of molecular gas is still available in the host. In addition, although requiring confirmation, the SFE may not yet have reached a level as high as seen in other, possibly more evolved, quasars. Measuring the flux density at $\sim 1 \mathrm{~mm}$ would help to better measure the IR luminosity and host SFR of $\mathrm{J} 0015+1842$ and similar sources.

If, as suggested by Noterdaeme et al. (2021), the presence of proximate $\mathrm{H}_{2}$ absorbers and leaking Ly- $\alpha$ emission provides an efficient way of identifying multi-phase outflows in regular quasars, then observations of $\mathrm{CO}$ emission in a sample of them could bring further clues to constrain the relative importance of orientation and evolutionary sequence in the appearance of quasars. Deep observations at high angular resolution are expected to allow confirming or refuting the derived configurations and enable detailed investigation of early feedback mechanisms in more regular quasars than those usually targeted for millimetre studies.

Acknowledgements. We thank the referee for insightful comments that helped improving the presentation of our results. We warmly thank Manuela Bischetti for kindly proving us with her compilation of CO and IR measurements for AGNs and SMGs and Pierre Cox for advises about NOEMA observations.

\footnotetext{
$5 L_{\mathrm{Edd}}=1.4 \times 10^{38}\left(M_{\mathrm{BH}} / M_{\odot}\right)$ and $\log M_{\mathrm{BH}} / M_{\odot} \sim 8.6-8.9$ is obtained from the width of the $\mathrm{CIV}$ (or $\mathrm{H}-\beta$ ) line and the rest-frame continuum luminosity at $1350 \AA$ ( $\mathrm{H}-\beta$ luminosity) using the calibration by Vestergaard \& Peterson (2006).
}

This work is based on observations carried out under project number S20BZ (PI Noterdaeme) with the IRAM NOEMA Interferometer. IRAM is supported by INSU/CNRS (France), MPG (Germany) and IGN (Spain). We are grateful to the IRAM staff for help with the data processing. The research leading to these results has received support from the French Agence Nationale de la Recherche under ANR grant 17-CE31-0011-01/project "HIH2" and from the French-Russian Collaborative Programme 1845. SB was supported by RSF grant 18-12-00301. The Cosmic Dawn Center is funded by the Danish National Research Foundation under grant No. 140.

\section{References}

Antonucci, R. 1993, ARA\&A, 31, 473

Banerji, M., Carilli, C. L., Jones, G., et al. 2017, MNRAS, 465, 4390 Banerji, M., Jones, G. C., Carniani, S., DeGraf, C., \& Wagg, J. 2021, MNRAS, 503, 5583

Barvainis, R., Maloney, P., Antonucci, R., \& Alloin, D. 1997, ApJ, 484, 695

Bertoldi, F., Cox, P., Neri, R., et al. 2003, A\&A, 409, L47

Bischetti, M., Piconcelli, E., Vietri, G., et al. 2017, A\&A, 598, A122

Bischetti, M., Feruglio, C., Piconcelli, E., et al. 2021, A\&A, 645, A33

Bolatto, A. D., Wolfire, M., \& Leroy, A. K. 2013, ARA\&A, 51, 207

Brusa, M., Feruglio, C., Cresci, G., et al. 2015, A\&A, 578, A11

Carilli, C. L., \& Walter, F. 2013, ARA\&A, 51, 105

Carniani, S., Marconi, A., Maiolino, R., et al. 2017, A\&A, 605, A105

Circosta, C., Mainieri, V., Lamperti, I., et al. 2021, A\&A, 646, A96

Coppin, K. E. K., Swinbank, A. M., Neri, R., et al. 2008, MNRAS, 389, 45

Drake, A. J., Djorgovski, S. G., Mahabal, A., et al. 2009, ApJ, 696, 870

Fabian, A. C. 2012, ARA\&A, 50, 455

Fan, L., Knudsen, K. K., Fogasy, J., \& Drouart, G. 2018, ApJ, 856, L5

Fan, L., Knudsen, K. K., Han, Y., \& Tan, Q.-H. 2019, ApJ, 887, 74

Feruglio, C., Bongiorno, A., Fiore, F., et al. 2014, A\&A, 565, A91

Gkini, A., Plionis, M., Chira, M., \& Koulouridis, E. 2021, A\&A, 650, A75

Heckman, T. M., Kauffmann, G., Brinchmann, J., et al. 2004, ApJ, 613, 109

Hook, I. M., McMahon, R. G., Boyle, B. J., \& Irwin, M. J. 1994, MNRAS, 268, 305

Hopkins, P. F., Hernquist, L., Cox, T. J., \& Kereš, D. 2008, ApJS, 175, 356

Kakkad, D., Mainieri, V., Brusa, M., et al. 2017, MNRAS, 468, 4205

Lewis, G. F., Carilli, C., Papadopoulos, P., \& Ivison, R. J. 2002, MNRAS, 330, L15

Miki, Y., Mori, M., \& Kawaguchi, T. 2021, Nat. Astron., 5, 478

Neri, R., Cox, P., Omont, A., et al. 2020, A\&A, 635, A7

Nesvadba, N. P. H., Polletta, M., Lehnert, M. D., et al. 2011, MNRAS, 415, 2359

Nesvadba, N. P. H., Bicknell, G. V., Mukherjee, D., \& Wagner, A. Y. 2020, A\&A, 639, L13

Noterdaeme, P., Balashev, S., Krogager, J. K., et al. 2019, A\&A, 627, A32

Noterdaeme, P., Balashev, S., Krogager, J. K., et al. 2021, A\&A, 646, A108

Omont, A., Petitjean, P., Guilloteau, S., et al. 1996, Nature, 382, 428

Perna, M., Sargent, M. T., Brusa, M., et al. 2018, A\&A, 619, A90

Polletta, M., Tajer, M., Maraschi, L., et al. 2007, ApJ, 663, 81

Polletta, M., Nesvadba, N. P. H., Neri, R., et al. 2011, A\&A, 533, A20

Pontzen, A., Tremmel, M., Roth, N., et al. 2017, MNRAS, 465, 547

Puglisi, A., Daddi, E., Brusa, M., et al. 2021, Nat. Astron., 5, 319

Rangwala, N., Maloney, P. R., Glenn, J., et al. 2011, ApJ, 743, 94

Riechers, D. A., Walter, F., Carilli, C. L., et al. 2006, ApJ, 650, 604

Salomé, P., Guélin, M., Downes, D., et al. 2012, A\&A, 545, A57

Sargent, M. T., Daddi, E., Béthermin, M., et al. 2014, ApJ, 793, 19

Sharon, C. E., Riechers, D. A., Hodge, J., et al. 2016, ApJ, 827, 18

Silk, J., \& Rees, M. J. 1998, A\&A, 331, L1

Simpson, J. M., Smail, I., Swinbank, A. M., et al. 2012, MNRAS, 426, 3201

Solomon, P. M., \& Vanden Bout, P. A. 2005, ARA\&A, 43, 677

Springel, V., Di Matteo, T., \& Hernquist, L. 2005, ApJ, 620, L79

Terrazas, B. A., Bell, E. F., Pillepich, A., et al. 2020, MNRAS, 493, 1888

Veilleux, S., Bolatto, A., Tombesi, F., et al. 2017, ApJ, 843, 18

Venemans, B. P., Walter, F., Decarli, R., et al. 2017, ApJ, 845, 154

Vestergaard, M., \& Peterson, B. M. 2006, ApJ, 641, 689

Volonteri, M., Haardt, F., \& Madau, P. 2003, ApJ, 582, 559

Walter, F., Bertoldi, F., Carilli, C., et al. 2003, Nature, 424, 406

Wang, R., Carilli, C. L., Neri, R., et al. 2010, ApJ, 714, 699

Wang, R., Wagg, J., Carilli, C. L., et al. 2013, ApJ, 773, 44

Wang, R., Wu, X.-B., Neri, R., et al. 2016, ApJ, 830, 53

Weiß, A., Downes, D., Neri, R., et al. 2007, A\&A, 467, 955

Weiß, A., Walter, F., Downes, D., et al. 2012, ApJ, 753, 102

Willott, C. J., Martínez-Sansigre, A., \& Rawlings, S. 2007, AJ, 133, 564

Wright, E. L., Eisenhardt, P. R. M., Mainzer, A. K., et al. 2010, AJ, 140, 1868

York, D. G., Adelman, J., Anderson, J. E., Jr, et al. 2000, AJ, 120, 1579

Zubovas, K., \& King, A. 2012, ApJ, 745, L34 